\section{Neue Luftreiniger mit Format}

\begin{abstract}
llergenkarenz ist neben der spezifischen Immuntherapie die einzige kausale Therapieoption bei einer allergischen Atemwegserkrankung - allein sie ist bei Aeroallergenen oft nur schwer zu realisieren. Effektive Unterstützung bei dem Versuch, Pollen, Tierhaare, Milbenkot oder Schimmelpilzsporen aus der Innenraumluft zu entfernen, versprechen die beiden PlasmaclusterLuftreiniger KC-860E und KC-850EW des Unternehmens Sharp. Neben der Filterfunktion sorgen sie auch für angenehme Luftfeuchtigkeit und verbessern durch ihre neuartige Plasmacluster-Ionen-Technologie die Luftqualität.

Das Filtersystem besteht aus drei Stufen: Bereits im ersten Schritt bleiben Hausstaub, Pollen und Schwebeteilchen an der Oberfläche des feinmaschigen Vorfilters und des Desodorierungsfilters hängen, unangenehme Gerüche werden dabei neutralisiert. Kleinere Staubpartikel bindet im zweiten Schritt ein HEPA-Filter, der 99,97\% aller Schwebeteilchen bis zu einer Größe von $0,3 \mu \mathrm{m}$ aus der durchströmenden Luft entfernt.

Der eigentliche Clou ist aber der Plasmacluster-Ionengenerator: Er spaltet die in der Raumluft enthaltenen Wasser-
\end{abstract}

moleküle in Wasserstoff und Sauerstoff auf. Tausende dieser Ionen werden anschließend an die Raumluft abgegeben und zersetzen unangenehme Gerüche und beseitigen verschiedene Schimmelpilzsporen. Die integrierte Raumluftbefeuchtungsfunktion mit Sprühnebeleffekt unterstützt diesen Prozess.

Komfortabel ist der Auto-Modus der Geräte: Bei dieser Betriebsart wird die Qualität der Raumluft durch Sensoren ständig erfasst und die erforderliche Reinigungsstufe samt Luftbefeuchtung kontinuierlich angepasst. Um die Raumluft immer so sauber wie möglich zu halten, sollten die Luftreiniger täglich 24 Stunden im Betrieb sein. Durch die integrierte Inverter-Technologie laufen die Geräte auf niedrigster Betriebsstufe sehr energiesparend und erfüllen mit einem Betriebsgeräusch von $17 \mathrm{~dB}$ (KC-860E) beziehungsweise $15 \mathrm{~dB}$ (KC-850EW) ihre Aufgabe nahezu geräuschlos. Zusätzlich ist die Displaybeleuchtung abschaltbar. So eignen sich die Luftreiniger auch für den Nachtbetrieb im Schlafzimmer.

Die Plasmacluster-Luftreiniger sind für Räume bis zu $48 \mathrm{~m}^{2}$ (KC-860E) beziehungsweise $38 \mathrm{~m}^{2}$ (KC-850EW) konzipiert. Weitere Informationen zu den

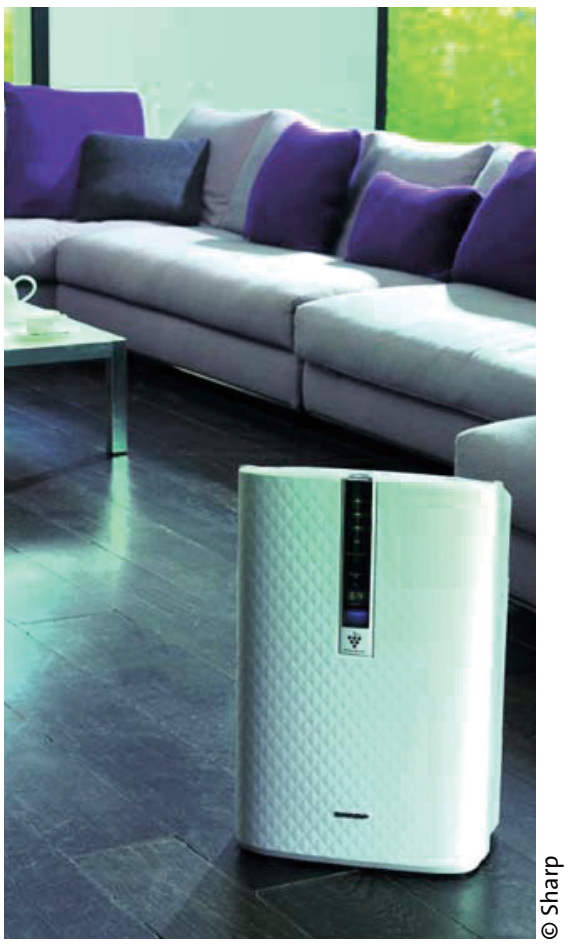

Moderne Luftreiniger versprechen Unterstützung bei dem Versuch, Allergene aus der Raumluft zu entfernen, und sind dabei auch dekorativ.

beiden Geräten sind unter www.sharp.de online abrufbar.

Nach Informationen von Sharp

Electronics, Hamburg

\title{
Neues Antihistaminikum ante portas
}

A ntihistaminika gehören zu den Mitteln der ersten Wahl bei der Behandlung der allergischen Rhinitis und der Urtikaria. Mit Bilastin wird demnächst eine neuer Vertreter aus der Gruppe der nicht sedierenden Antihistaminika in Europa erhältlich sein, erklärte Prof. Dr. Claus Bachert, Gent, Belgien. Die Substanz bindet selektiv an den H1-Rezeptor, hat keine ZNS-Effekte und zeigt selbst bei sehr hohen Dosen ein gutes kardiales Sicherheitsprofil. Bilastin wird nicht metabolisiert und unverändert über Urin und Faeces ausgeschieden. Interaktionen mit Alkohol oder sedierenden Arzneimitteln treten nicht auf.

In klinischen Studie mit Patienten mit einer allergischen Rhinokonjunktivi- tis ergab sich unter $20 \mathrm{mg}$ Bilastin einmal täglich eine vergleichbare therapeutische Effizienz wie unter anderen Antihistaminika der zweiten Generation, berichtete Bachert. Die Wirkung von Bilastin tritt rasch ein, Nebenwirkungen sind sehr selten und in der Regel mild.

In der Behandlung der Urtikaria können Antihistaminika bis zum Vierfachen der empfohlenen Tagesdosis gegeben werden, wobei den Antihistaminika der zweiten Generation leitliniengerecht der Vorzug zu geben ist, referierte Prof. Dr. Torsten Zuberbier, Berlin. Die Antihistaminika der zweiten Generation sind nicht sedierend und verfügen neben ihrer H1-Rezeptor-blockierenden Wirkung über zu- sätzliche Effekt, etwa auf die Zytokinausschüttung sowie mastzellabhängige Reaktionsabläufe. In der Behandlung der chronischen Urtikaria sind $20 \mathrm{mg}$ Bilastin einmal täglich vergleichbar sicher und effektiv wie $5 \mathrm{mg}$ Levocetirizin.

Zur Beurteilung des Stellenwerts des neuen Antihistaminikums müssten nun große Studien folgen, so die Referenten, in denen vor allem das gute Verträglichkeitsprofil von Bilastin herausgearbeitet werden sollte.

$b k$

Satellitensymposium „Developing future in antihistamines“ im Rahmen des 29. Kongresses der European Academy of Allergy and Clinical Immunology (EAACI). London, Großbritannien, 6. Juni 2010. Veranstalter: Faes Farma, Leioa, Spanien 